\title{
Laparoscopic versus open groin hernia repair: are we getting closer to specific clinical recommendations?
}

\author{
H. Kehlet
}

Received: 14 September 2010 / Accepted: 3 October 2010 / Published online: 16 October 2010

(c) Springer-Verlag 2010

The choice between a laparoscopic versus an open mesh repair for a groin hernia has been debated for years and the current evidence-based guidelines from the European Hernia Society recommend that both techniques can be used, but that a laparoscopic repair is followed by less acute postoperative pain, a shorter convalescence and, potentially, a lower risk of chronic pain, while there is no difference in the risk of a recurrence [1]. Since there may be a risk of rare but serious intra-abdominal complications with the laparoscopic repair and since the intra-operative costs may be higher, as well as the demand for expertise, there is still a need for more well-defined recommendations for groin hernia surgery regarding the type of procedure and on who is going to perform the operation and in which patients?

Although the evidence from plenty of randomised trials suggests that the acute pain intensity is less with a laparoscopic repair, the problem with all of these studies is that little attention has been paid to treating the acute pain after an open repair by simple local and multi-modal oral analgesics [2]. Thus, the current evidence-based post-operative pain management consists of multiple non-opioid analgesics and, by such treatment, the difference in acute pain responses may probably be minor between the two techniques, except in the minority of patients where optimal dynamic pain relief, i.e. analgesia during exercise or heavy work, is required. The same considerations exist about the quality of convalescence data from the randomised trials comparing the two techniques, since they have rarely

H. Kehlet $(\square)$

Section for Surgical Pathophysiology,

Rigshospitalet Copenhagen University,

Copenhagen, Denmark

e-mail: henrik.kehlet@rh.dk focused on specific groups of patients in relation to the type of exercise. Thus, such detailed studies have shown a pronounced difference between patient groups and with very short (less than 1 week) convalescence after an open Lichtenstein repair in those patients with less demanding physical function and work [3]. Therefore, combining the data from acute pain and convalescence where elderly patients have a reduced pain response to surgery and a generally lower activity may support the use of the more easily performed and less costly open mesh repair in such patients.

Nevertheless, there is now an agreement between most groin hernia surgeons that the risk of chronic pain complaints represents the most important outcome of groin hernia repair [4], given that recurrence rates are so low with the current two major techniques. Although much data on chronic pain after laparoscopic versus an open mesh repair are available [1], the problem with most of these studies is that they have been designed primarily to assess the risk of a recurrence and with chronic pain as a secondary outcome and most often assessed sub-optimally and not in relation to specific daily activities or well-described characteristics and intensity [5]. Furthermore, although chronic post-herniorrhaphy pain has been considered mostly to be of neuropathic origin [6], the documentation for neuropathic versus chronic inflammatory pain has been extremely scarce [6]. However, recently, a large two-centre prospective study has been published among 442 patients, with, for the first time, a detailed description of all pre-, intra- and post-operative risk factors for the development of persistent post-herniotomy pain [7]. In this study, two high-volume centres were chosen (one in Copenhagen and one in Stuttgart), each performing either a well-established Lichtenstein repair or a laparoscopic transabdominal pre-peritoneal (TAPP) repair. The results with detailed assessment of neurophysiological function in the surgical area, characteristics and intensity 
and social consequences [5] of the persistent pain showed, as with most of the other studies [1], that there was about $50 \%$ less risk of persistent pain after the laparoscopic repair, and with a lower intensity. The detailed neurophysiological assessments showed, for the first time, less sign of nerve damage with the laparoscopic mesh repair with glue fixation [7]. However, probably the most important finding of this study was that the pre-operative function of the nociceptive system, i.e. the pain response to a 5 -s $47^{\circ} \mathrm{C}$ heat stimulus, was of equal importance as to the type of surgery to predict the risk of chronic pain. From a multiple logistic regression analysis of pre- and intra-operative risk factors, it was concluded that, in pre-operative high pain responders to heat stimulation, there was an even more pronounced reduction of the risk of persistent pain with the laparoscopic repair compared with the open repair. In contrast, in pre-operative low pain responders, there was no difference in the chronic pain outcome between the two techniques. These results, which obviously need to be confirmed, suggest that we, in the future, may have a more scientific basis for choosing the optimal groin hernia repair technique in that pre-operative low pain responders should be treated with the simple and cheap open mesh repair, while pre-operative high pain responders should be allocated for a laparoscopic repair in an expert centre.

What, then, are the future challenges in groin hernia repair regarding the risk of persistent pain [4]? First of all, there is agreement that this is the most important outcome, that the provision of surgical expertise in both techniques will be required, that specific attention should be paid towards avoiding nerve injury, including the fixation technique of the mesh, and where glue fixation should be considered and studied in more detail, as well as newer lightweight mesh modalities [4]. Secondly, simple, cheap assessment of nociceptive function, e.g. by the pain response to a heat stimulus, may help us to achieve better patient selection and where low pain responders should receive an open mesh repair under local anaesthesia being most costeffective repair and with smaller risk of morbidity in elderly patients [8]. Finally, future studies are required on acute pain and convalescence between the two techniques and including optimal evidence-based multi-modal oral analgesia [2], as well as stratification into relevant sub-groups of work intensity [3] and short well-defined convalescence recommendations.

In conclusion, the improved quality of research in groin hernia repair during the last decade has undoubtedly helped us in the better selection of patients and surgeons to a more rational choice of surgical technique between open and laparoscopic repairs. The more recent research on the role of individual patient factors for the development of persistent pain may, hopefully, lead to further answers within the next few years.

Conflict of interest None.

\section{References}

1. Simons MP, Aufenacker T, Bay-Nielsen M, Bouillot JL, Campanelli G, Conze J, de Lange D, Fortelny R, Heikkinen T, Kingsnorth A, Kukleta J, Morales-Conde S, Nordin P, Schumpelick V, Smedberg S, Smietanski M, Weber G, Miserez M (2009) European Hernia Society guidelines on the treatment of inguinal hernia in adult patients. Hernia 13:343-403

2. White PF, Kehlet H (2010) Improving postoperative pain management: what are the unresolved issues? Anesthesiology 112:220-225

3. Bay-Nielsen M, Thomsen H, Andersen FH, Bendix JH, Sørensen OK, Skovgaard N, Kehlet H (2004) Convalescence after inguinal herniorrhaphy. Br J Surg 91:362-367

4. Kehlet H (2008) Chronic pain after groin hernia repair. Br J Surg 95:135-136

5. McCarthy M Jr, Jonasson O, Chang CH, Pickard AS, GiobbieHurder A, Gibbs J, Edelman P, Fitzgibbons R, Neumayer L (2005) Assessment of patient functional status after surgery. J Am Coll Surg 201:171-178

6. Kehlet H, Jensen TS, Woolf CJ (2006) Persistent postsurgical pain: risk factors and prevention. Lancet 367:1618-1625

7. Aasvang EK, Gmähle E, Hansen JB, Gmähle B, Forman JL, Schwarz J, Bittner R, Kehlet H (2010) Predictive risk factors for persistent postherniotomy pain. Anesthesiology 112:957-969

8. Bay-Nielsen M, Kehlet H (2008) Anaesthesia and post-operative morbidity after elective groin hernia repair: a nation-wide study. Acta Anaesthesiol Scand 52:169-174 